\title{
A PUZZLING HEPATIC TUMOR: EPITHELIOID ANGIOMYOLIPOMA WITH A MINOR ADIPOCYTIC COMPONENT
}

\author{
F. Limaiem ${ }^{1}$, Seifeddine BACCOUCH ${ }^{2}$, Nadia BOUJELBENE ${ }^{3}$, Aziz ATALLAH ${ }^{2}$, \\ Mohamed HAJRI ${ }^{2}$, Sofiene GABSI ${ }^{2}$, Leila Ben Farhat ${ }^{2}$, and saadia bouraoui ${ }^{4}$ \\ ${ }^{1}$ Univ Tunis El Manar \\ ${ }^{2}$ CHU Mongi Slim \\ ${ }^{3}$ Institut Salah-Azaiz \\ ${ }^{4}$ University Hospital Center Mongi Slim
}

September 25, 2021

\begin{abstract}
Hepatic epithelioid angiomyolipomas are uncommon mesenchymal neoplasms. They are difficult to diagnose by imaging, especially when the fatty component is scant or absent. The gold standard for the diagnosis is histologic examination coupled with an immunohistochemical study. Positive HMB-45 immunostaining of the myoid cells is a major diagnostic feature.
\end{abstract}

\section{INTRODUCTION}

Hepatic angiomyolipomas are rare mesenchymal neoplasms composed of an admixture of smooth muscle cells, adipose tissue, and blood vessels [1]. Epithelioid angiomyolipoma is a rare variant of angiomyolipoma where the epithelioid smooth muscle cells predominate [2]. Due to their varying composition, particularly their frequent paucity of fat, diagnosis can be challenging both radiologically and histologically resulting in misdiagnosis prior to excision [3]. Herein, the authors report a new case of hepatic epithelioid angiomyolipoma with a minor adipocytic component mimicking a hepatocellular carcinoma. They describe its histopathological characteristics illustrating the wide variety of its appearances, and highlight the challenges and potential errors that can be made.

\section{CLINICAL HISTORY}

A 37-year-old previously healthy woman, presented with a five-month history of weight loss anorexia, abdominal distention and discomfort. The patient had no prior history of hepato-biliary disease or tuberous sclerosis. Physical examination disclosed hepatomegaly with a liver span equal to $20 \mathrm{~cm}$ (Figure $1 \mathrm{~A}$ ). On palpation of the abdomen, there was a renitent firm mass in the upper right quadrant mobile with respect to the superficial planes and fixed with respect to the deep planes. Tumor markers, including alpha-fetoprotein, carcinoembryonic antigen, and carbohydrate antigen 19-9 were all within normal limits. Aspartate aminotransferase, alanine aminotransferase and total bilirubin were within the normal range. Contrast-enhanced computed tomography scan demonstrated a hepatic mass located in segments IV, V and VI, with exophytic development, moderate and heterogeneous arterial enhancement, continuing in the portal and late stages, without detectable washout or late capsular enhancement (Figures 1B \& 1C). This mass measured $170 \mathrm{x}$ $110 \mathrm{~mm}$ in the axial plane and $200 \mathrm{~mm}$ in height. It had a close vascular contact with the right hepatic artery, the cystic artery, the right portal branch as well as its segmental portal branches of IV and VI, which remained permeable, without real invasion. Computed tomography scan also disclosed a left ovarian mass 
measuring $90 \times 60 \times 90 \mathrm{~mm}$ suggestive of teratoma (Figure 1C). On abdominal magnetic resonance imaging (MRI), low intensity was observed on a T1-weighted image and high intensity on a T2-weighted image. The patient underwent fine needle biopsy of the hepatic mass and histopathological examination of the biopsy specimen was suggestive of hepatocellular carcinoma. Right hepatectomy was performed with informed consent of the patient. Intraoperatively, a relatively soft dark red giant tumor was found to occupy the whole right lobe of the liver (Figure 1D). We received a hepatectomy specimen which weighed 1050 grams and measured after formalin fixation $17 \times 15.5 \times 6.5 \mathrm{~cm}$. Cut section showed a well-circumscribed cystic mass measuring $16 \mathrm{~cm}$ in diameter with a hemorrhagic appearance and focal grey white firm areas in the periphery (Figure 2A). Histologically, the mass was composed of an admixture of smooth muscle cells, adipose tissue, and blood vessels (Figure 2B). Sheets of mature adipocytes were seen in some of the sections focally. The tumor proliferation consisted of $100 \%$ of epithelioid cells arranged in a trabecular pattern (Figure 2C) or in sheets. Extensive extramedullary hematopoiesis was noted in several sections with hematopoietic elements, including megakaryocytes as well as erythroid and myeloid precursors (Figure 2D). The vascular component was abundant and was composed of thick (Figure 2B) and thin-walled vessels with a striking peliotic pattern in some areas (Figure 3A). The epithelioid cells showed round nuclei, and abundant eosinophilic or clear cytoplasm (Figure 3B). Brown melanin granules were focally found within the tumor (Figure $3 \mathrm{C}$ ). Neither tumor necrosis nor mitotic figures were observed. The nonneoplastic liver showed mild nonspecific mononuclear infiltration in the portal tracts. Based on these microscopic findings, epithelioid angoimyolipoma, epithelioid hemangioendothelioma, hepatocellular carcinoma and hepatocellular adenoma were considered as differential diagnoses and an immunohistochemical study was performed. HMB45 (Figure 3D), MelanA, Smooth Muscle Actin and Desmin showed diffuse strong positive staining. However, the tumor cells showed negative staining for anti-hepatocyte, and CD34. The postoperative course was uneventful, and the patient was discharged on postoperative day 10. At present, she is still being followed-up.

\section{DISCUSSION}

Epithelioid angiomyolipoma is an uncommon variant of angiomyolipoma where the epithelioid smooth muscle cells predominate and is classified as a perivascular epithelioid cell tumor [4]. Epithelioid angiomyolipoma occurs most commonly in the kidneys and rarely involves the liver. More than 200 cases of hepatic angiomyolipomas have been reported in the English language literature so far. The age at diagnosis ranges from 10 to 80 years. Cases in children are rare [5]. Our patient was 37 years old. In contrast to renal angiomyolipomas, which are associated with tuberous sclerosis in up to $20 \%$ of patients, hepatic angiomyolipomas are associated with tuberous sclerosis in only $6 \%$ of patients, with a female predominance [6,7]. Our patient had no past medical history of tuberous sclerosis. Most patients with hepatic angiomyolipomas are asymptomatic, and their tumors are found incidentally during routine health check-ups $[1,5,6]$. In a small proportion of cases, patients may have mass compression effects including epigastric intermittent pain, nausea, and dyspepsia [1,5-7]. Our patient presented with a five-month history of weight loss, anorexia, abdominal distention and discomfort. There are no helpful laboratory tests for this disease. The liver function is normal, tumor and hepatitis markers are negative as it was the case in our patient. The imaging appearance of hepatic epithelioid angiomyolipoma is variable since the relative proportion of tissue components varies widely. Imaging studies are not necessarily adding diagnostic value, especially in tumors with minimal fat component [8]. In our patient, the imaging findings were not suggestive of hepatic angiomyolipoma since the adipocytic component was inconspicuous. Contrast enhancement on arterial phase and hypoattenuation/hypointensity on portal phase were a common finding on imaging for epithelioid angiomyolipoma of the liver [9]. Even with the advances of nuclear medicine technologies, in the presence of hemorrhage and associated inflammatory response, hepatic angiomyolipomas may present with increased fluorodeoxyglucose uptake on imaging studies [10]. Macroscopically, hepatic angiomyolipomas with significant fat content tend to be soft and yellow, whereas tumors with predominantly smooth muscle content tend to be firm, with a tan-white cut surface. Necrosis and hemorrhage may occur. Cystic appearance with hemorrhagic areas is rare which added to the diagnostic difficulty in our case $[1,11]$. Histologically, hepatic angiomyolipomas resemble their counterpart in the kidney. They contain a variable amount of fat, vessels, and smooth muscle cells $[1,6,7,11]$. Four types of smooth muscle cell can be found histologically within angiomyolipomas including spindle, intermediate, 
epithelioid and pleomorphic cells [6]. The percentage of epithelioid cells that is required to make a diagnosis of epithelioid angiomyolipoma was not defined in the published studies. A study by Aydin et al. proposed that using $10 \%$ of epithelioid component as the cut-off value was preferred [11]. In our patient, $100 \%$ of the tumor cells were epithelioid. The vascular component is typically made of thick-walled hyalinized arterial or venous-like vessels with abnormally thickened hyperplastic elastic fibers as it was the case in our patient $[1,6]$. Foam cells with fine lipid droplets and brown melanin granules can be seen. In our case, brown melanin granules were focally found within the tumor. Depending on the proportions of the three tissue elements in angiomyolipoma, it can be classified into 10 types; mixed (conventional), myomatous, lipomatous, angiomatous, myoangiomatous, angiomyomatous, myolipomatous, lipomyomatous, lipoangiomatous, and angiolipomatous [1]. These subtypes have no clinical or prognostic significance. Awareness of these types will be useful in understanding and interpreting a wide range of imaging findings for hepatic angiomyolipomas. In our patient, the tumor is classified as a mixed epithelioid angiomyolipoma with a minor adipocytic component. Hepatic angiomyolipoma often contains hematopoietic elements, including megakaryocytes as well as erythroid and myeloid precursors. In our case, there were extensive foci of extra-medullary hematopoiesis. The pathognomonic feature of angiomyolipoma is the positivity of smooth muscle cells for human melanoma black-45 (HMB-45) along with the positivity for muscle markers. The expression of melanocytic and muscle markers places this tumor in the category of PEComas. CD117 (c-kit) and tyrosinase are also positive in angiomyolipomas [12,13]. In our patient, HMB45, MelanA, Smooth Muscle Actin and Desmin showed diffuse strong positive staining. However, the tumor cells showed negative staining for anti-hepatocyte, and CD34.

The main differential diagnosis of epithelioid angiomyolipoma is hepatocellular carcinoma or hepatocellular adenoma [1,14]. Many features of hepatic angiomyolipoma can lead to a misdiagnosis of hepatocellular carcinoma, such as polygonal cells in a trabecular arrangement, nuclear pleomorphism, eosinophilic globules, and tumor necrosis. However, hepatocellular carcinomas usually express HepPar 1 and do not express smooth muscle or melanoma antigens $[1,14]$. In our case, the diagnosis of hepatocellular carcinoma was suggested on fine needle biopsy because of the epitheliod morphology of tumor cells which were arranged in a trabecular pattern. Tumors with a predominant fat component may be mistaken for focal fatty change or hepatocellular adenoma. The combination of spindle and epithelioid cells and positivity for HMB-45 may mimic metastatic malignant melanoma [1,14]. Furthermore, when epithelioid smooth muscle cells with clear cytoplasm arranged in a solid or alveolar pattern are encountered, the possibility of metastatic renal cell carcinoma must be excluded.

Previous studies suggested that hepatic epithelioid angiomyolipoma can be managed by conservative treatment with follow-up [15]. Surgical intervention may be needed in selected patients with symptomatic masses to alleviate effects on neighboring organs [16]. Hepatic epithelioid angiomyolipoma is regarded as a tumor of uncertain behavior; cases of epithelioid angiomyolipoma with local recurrence and multiple metastases have been reported [16].

In conclusion, hepatic epithelioid angiomyolipomas can lead to considerable diagnostic problems clinically, radiologically, and pathologically because of their diverse morphology. Diagnostic confusion arises when the fat cell component is inconspicuous and the smooth muscle component assumes an unusual morphologic phenotype. The diagnostic difficulty escalates in a fine needle aspiration setting. The striking feature in our case was the unusual macroscopic appearance of the tumor which was cystic with hemorrhage as well as the prominence of extramedullary hematopoiesis. Positive HMB-45 immunostaining of the myoid cells is a major diagnostic feature. The possibility of angiomyolipoma should be considered whenever unfamiliar hepatic tumors are encountered.

\section{CONFLICT OF INTEREST STATEMENT}

None declared.

\section{AUTHORS' CONTRIBUTIONS:}

Dr Faten LIMAIEM, Dr Nadia BOUJELBENE and Pr Saadia BOURAOUI prepared, organized, wrote, and edited all aspects of the manuscript. They performed the gross and microscopic pathologic 
evaluation of the pathology specimen. They prepared all of the histology figures in the manuscript. They read, edited, and approved the final version of the manuscript.

Dr Seifeddine BACCOUCH, Dr Aziz ATALlAH, Dr Mohamed HAJRI, Dr Sofiène GABSI and Leila BEN FARHAT participated in:

1. The conception and design of the study,

2. The acquisition of data, analysis and interpretation of the data,

3. The drafting of the article and revising it critically for important intellectual content,

4. Final approval of the manuscript before its submission

\section{ETHICAL APPROVAL}

All procedures performed were in accordance with the ethical standards. The examination was made in accordance with the approved principles.

\section{ACKNOWLEDGEMENT STATEMENT}

I would like to offer my special thanks to Mrs Saïda Jerbi who prepared the microscopic slides. Published with written consent of the patient.

\section{DATA AVAILABILITY}

In accordance with the DFG Guidelines on the Handling of Research Data, we will make all data available upon request.

\section{FUNDING STATEMENT:}

This research did not receive specific funding

\section{REFERENCES}

[1] Tsui WMS, Colombari C, Portmann BC et al. Hepatic angiomyolipoma; a clinicopatholgic study of 30 cases and delineation of unusual morphologic variants. Am. Surg. Pathol. 1999; 23; 34-38.

[2] Nonomura A, Mizukami Y, Kadoya M, et al. Angiomyolipoma of the liver: its clinical and histological diversity. J. Hepat. Bil. Surg. 1996; 3; 122-132.

[3] Nonomura A, Mizukami Y, Muraoka K, et al. Angiomyolipoma of the liver with pleomorphic histologic features. Histopathology 1994; 24; 279-281

[4]. Maebayashi T, Abe K, Aizawa T et al. Improving recognition of hepatic perivascular epithelioid cell tumor: case report and literature review. World J Gastroenterol 2007; 21: 5432-41.

[5] Yeh CN, Chen MF, Hung TC, et al. Angiomyolipoma of the liver. J Surg Oncol 2001;77:195e200.

[6]. Nonomura A, Enomoto Y, Takeda M, et al. Angiomyolipoma of the liver: a reappraisal of morphological features and delineation of new characteristic histological features from the clinicopathological findings of 55 tumours in 47 patients. Histopathology. $2012 ; 61(5): 863-80$.

[7]. Goodman ZD, Ishak KG. Angiomyolipomas of the liver. Am J Surg Pathol 1984;8:745-750.

[8]. Lee SJ, Kim SY, Kim KW, et al. Hepatic angiomyolipoma with minimal fat, mimicking hepatocellular carcinoma. Clin Mol Hepatol 2012;18(3):330-5.

[9]. Xu PJ, Shan Y, Yan FH, et al. Epithelioid angiomyolipoma of the liver: cross-sectional imaging findings of 10 immunohistochemically-verified cases. World J Gastroenterol 2009;15(36):4576-81.

[10] Takanami K, Kaneta T, Hitachi S, et al. F-18 FDG PET/CT findings in two patients with hepatic angiomyolipoma with and without intratumoral hemorrhage. Clin Nucl Med 2010;35(1):18-21. 
[11] Aydin H, Magi-Galluzzi C, Lane B, et al. Renal angiomyolipoma: clinicopathologic study of 194 cases with emphasis on the epithelioid histology and tuberous sclerosis association. Am J Surg Pathol 2009;33:289e97.

[12] Nonomura A, Mizukami Y, Takayanagi N et al. Immunohistochemical study of hepatic angiomyolipoma. Pathol. Int. 1996; 48; 24-32.

[13] Ma TK, Tse MK, Tsui WM, et al. Fine needle aspiration diagnosis of angiomyolipoma of the liver using a cell block with immunohistochemical study. A case report. Acta Cytol 1994;38:257e60.

[14] Xie L, Jessurun J, Manivel JC, et al. Hepatic epithelioid angiomyolipoma with trabecular growth pattern: a mimic of hepatocellular carcinoma on fine needle aspiration cytology. Diagn Cytopathol 2012;40(7):639-50.

[15] Yang CY, Ho MC, Jeng YM, et al. Management of hepatic angiomyolipoma. J Gastrointest Surg 2007;11:452e7.

[16]. Deng Y, Lin Q, Zhang S, et al. Malignant angiomyolipoma in the liver: a case report with pathological and molecular analysis. Pathol Res Pract 2008;204:911e8.

FIGURE LEGENDS:

Multipanel Figure 1:

Figure 1 A: Physical examination disclosed hepatomegaly with an increased liver span $=20 \mathrm{~cm}$.

Figure 1 B: Axial Contrast-enhanced computed tomography scan demonstrated a hepatic mass (yellow asterisk) located in segments IV, V and VI, with exophytic development, moderate and heterogeneous arterial enhancement, continuing in the portal and late stages, without detectable washout or late capsular enhancement.

Figures 1 C: Coronal Computed tomography scan demonstrating a hepatic mass (blue arrow) located in segments IV, V and VI and a left ovarian mass suggestive of teratoma (white arrow).

Figure 1 D: Per-operative gross findings of the hepatic mass.

Multipanel Figure 2:

Figure 2 A: On gross examination (after formalin fixation), the cut surface of the liver showed a welldemarcated cystic mass measuring $16 \mathrm{~cm}$ in diameter with a hemorrhagic appearance and grey white firm areas in the periphery.

Figure 2 B: Histological findings of the hepatic tumor showing an admixture of sheets of epithelioid myoid cells, thick-walled abnormal blood vessels, and islands of mature adipocytes. The blood vessels are surrounded by mantles of epithelioid myoid cells (Hematoxylin and eosin staining, $\mathrm{x} 40$ ).

Figures 2 C: Tumor cells were arranged in a trabecular pattern( Hematoxylin and eosin, magnification $\times$ 200).

Figure 2 D: Some tumor cells exhibited clear or granular cytoplasm and were in relation with adipocytes. Note the extramedullary hematopoiesis ( Hematoxylin and eosin, magnification $\times 400$ ).

Multipanel Figure 3:

Figures 3 A: A striking peliotic pattern was focally noted( Hematoxylin and eosin, magnification $\times 40$ ).

Figures 3 B: The epithelioid myoid cells had bland vesicular nuclei and a clear cytoplasm ( Hematoxylin and eosin, magnification $\times 400$ ).

Figures 3 C: The epithelioid myoid cells had bland vesicular nuclei and eosinophilic cytoplasm. Note the melanin pigment( Hematoxylin and eosin, magnification $\times 400$ ).

Figures 3 D: Immunohistochemical study showing positive immunostaining of the tumor cells with the HMB45 antibody, (Immunohistochemistry, magnification $\times 400$ ). 

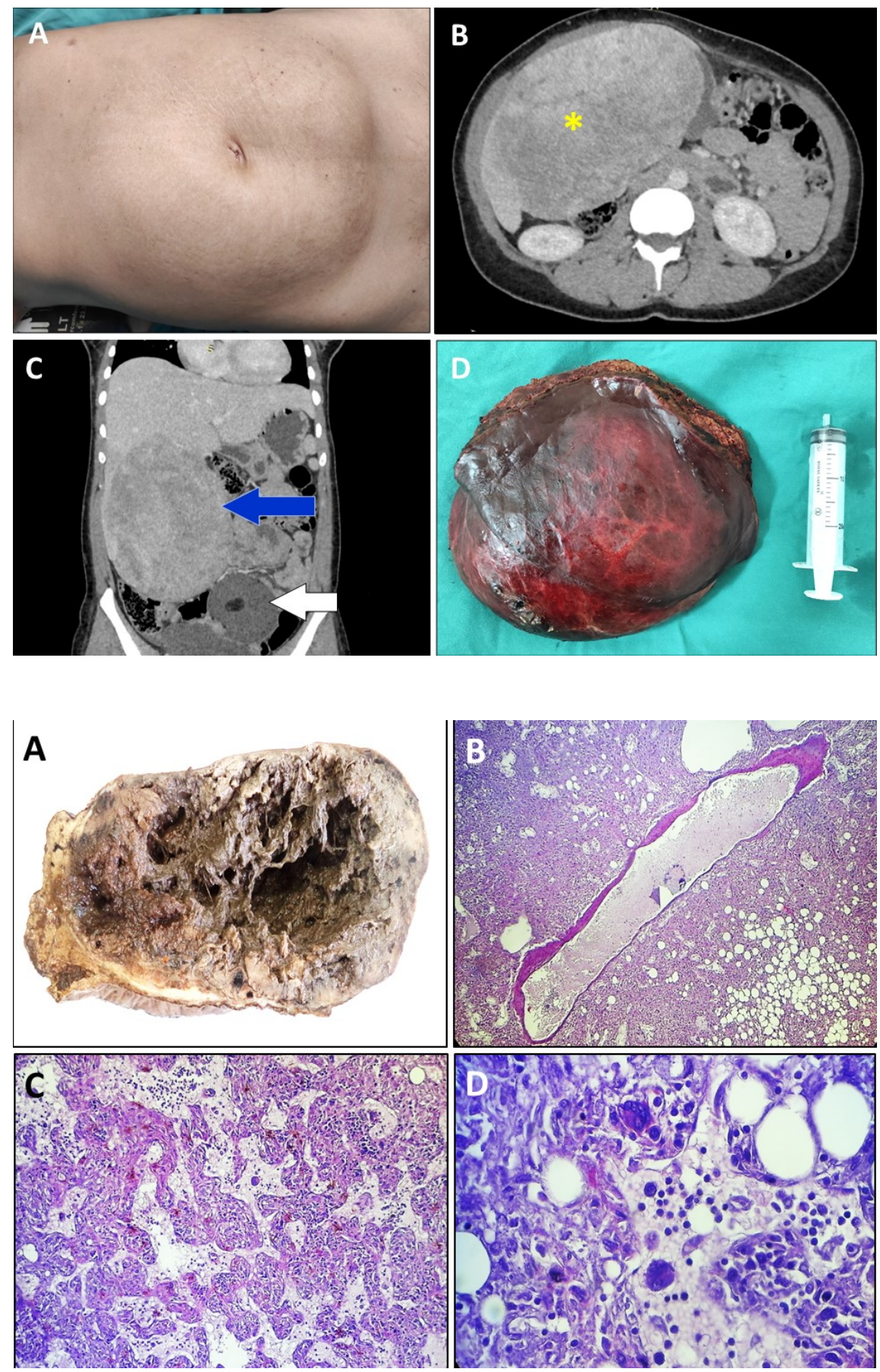


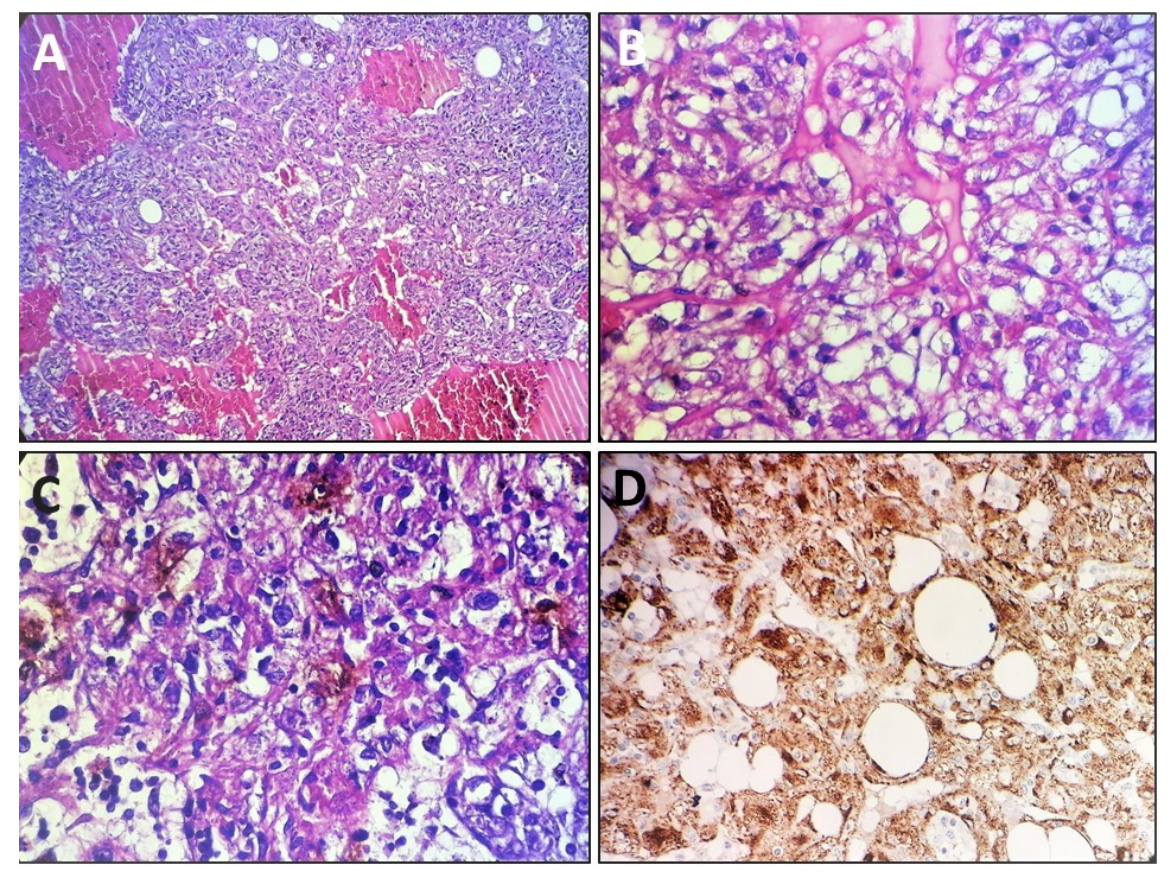

